\title{
Multiplicative properties of Morin maps
}

\author{
GÁBOR LIPPNER \\ ANDRÁS SZÜCS
}

\begin{abstract}
In the first part of the paper we construct a ring structure on the rational cobordism classes of Morin maps (ie smooth generic maps of corank 1). We show that associating to a Morin map its $\Sigma^{1 r}$ (or $A_{r}$ ) singular strata defines a ring homomorphism to $\Omega_{*} \otimes \mathbb{Q}$, the rational oriented cobordism ring. This is proved by analyzing the multiple-point sets of a product immersion. Using these homomorphisms we compute the ring of Morin maps.

In the second part of the paper we give a new method to find the oriented Thom polynomial of the $\Sigma^{2}$ singularity type with $\mathbb{Q}$ coefficients. Then we provide a product formula for the $\Sigma^{2}$ singularity in $\mathbb{Q}$ and the $\Sigma^{1,1}$ singularity in $\mathbb{Z}_{2}$ coefficients.
\end{abstract}

57R20, 57R42, 57R45

\section{Introduction}

The results of this paper are the first steps toward understanding how the direct product operation affects the singularities of maps. There are two main difficulties. The first one is that the direct product of generic maps will not be generic, so one has to take a small perturbation. This makes it hard to understand the singular strata geometrically. The second one is that in general the product of two singular maps even after a generic perturbation will have more complicated singularities then the original maps had.

In Section 2 we study products of immersions. Here only the first type of difficulty arises, namely that the self intersections will not be transverse. This can be overcome by employing a general multiple-point formula from Braun and Lippner [2] that helps to compute the characteristic numbers of multiple-point manifolds.

In Section 3 we study Morin maps. In this case we have to deal with the second kind of difficulty. Instead we get around it by increasing the dimension of the target space by one.

In Section 4 we set out to compute the ring $\operatorname{Mor}_{\mathbb{Q}}$ (the ring of rational cobordism classes of Morin maps) defined at the end of Section 3. First, in Section 4.1, we identify the components of Mor $_{\mathbb{Q}}$ as subgroups of the rational oriented cobordism ring $\Omega_{*} \otimes \mathbb{Q}$. 
Then combining the results of the previous sections we show that the singular strata behave nicely under the multiplication defined in Section 3.2. It turns out that this information is actually enough to compute $\operatorname{Mor}_{\mathbb{Q}}$.

Finally Section 5 deals with general singular maps. We show that a Cartan-type formula relates the homology class of $\Sigma^{1}$ points of two maps with that of their direct product. We give a new method of computing the oriented Thom polynomial of the $\Sigma^{2}$ singularity with $\mathbb{Q}$ coefficients, the value of which was already known (see Ronga [6]). Finally we derive a Cartan-type formula for the $\Sigma^{2}$ points as well.

This research was partially supported by grant T049449 of the Hungarian Scientific Research Fund.

\section{Products of immersions}

We start this section by recalling some basic notions about multiple points and the relevant results of [2].

First we shall introduce a characteristic class $\beta$ that assigns to any oriented vector bundle $\xi$ over $B$ an element

$$
\beta(\xi)=\prod_{i=1}^{\infty}\left(1+p_{1}(\xi) t_{i}+p_{2}(\xi) t_{i}^{2}+\cdots\right) \in H^{*}(B ; \mathbb{Q}) \llbracket t_{1}, t_{2}, \ldots \rrbracket
$$

in the ring of formal power series of the variables $t_{i}$ over the ring $H^{*}(B ; \mathbb{Q})$. (Here $p_{i}(\xi) \in H^{4 i}(B ; \mathbb{Q})$ is the $4 i$-dimensional Pontrjagin class of $\xi$.) Since the Cartan formula holds for Pontrjagin classes modulo 2-torsion it follows that $\beta(\xi \oplus \eta)=$ $\beta(\xi) \cdot \beta(\eta)$. (We have got rid of all torsion by taking $\mathbb{Q}$ coefficients.) It is also easily seen that $\beta$ is natural, and always has an inverse element. When $B$ is a manifold we shall abbreviate $\beta(T B)$ by $\beta(B)$.

Now let $f: M^{n} \rightarrow N^{n+k}$ be a generic (ie selftransverse) immersion between oriented manifolds. The manifolds and the maps representing the $r$-fold points of $f$ in the source and the target respectively will be denoted by

$$
\begin{aligned}
& \phi_{r}(f): \widetilde{M}_{r}(f) \rightarrow M, \\
& \psi_{r}(f): \tilde{N}_{r}(f) \rightarrow N .
\end{aligned}
$$

When the codimension of the map $k$ is even, these manifolds are equipped with natural orientations. It is easy to see that the cobordism classes of these manifolds depend only on the cobordism class of $f$. Our goal is to obtain information about these cobordism classes. To this end we compute their characteristic numbers. 
Let us introduce the notation

$$
\begin{aligned}
m_{r} & =m_{r}(f)=\phi_{r}(f)_{!}\left(\beta\left(\widetilde{M}_{r}(f)\right)\right), \\
n_{r} & =n_{r}(f)=\psi_{r}(f)_{!}\left(\beta\left(\tilde{N}_{r}(f)\right)\right) .
\end{aligned}
$$

The reason for considering these elements is the following simple observation. Evaluating each coefficient of $m_{r}$ on the fundamental class of $M$ we get an element in $\mathbb{Q} \llbracket t_{1}, t_{2}, \ldots \rrbracket$. The coefficients of this power series are exactly the Pontrjagin numbers of $\widetilde{M}_{r}(f)$.

The classes $m_{r}$ and $n_{r}$ are related by the equality

$$
m_{r} \cdot \beta\left(v_{f}\right)=f^{*} n_{r-1}-e\left(v_{f}\right) m_{r-1}
$$

where $v_{f}$ is the normal bundle of $f$ and $e$ is the Euler class. This is a generalization of the well-known Herbert-Ronga formula (see the main formula of [2]).

We are going to apply this in the case when the target is a Euclidean space. Then $f^{*}=0$ so (1) is simplified to $m_{r} \cdot \beta\left(v_{f}\right)=-e\left(v_{f}\right) \cdot m_{r-1}$. Applying this recursively one gets that $m_{r} \cdot \beta\left(v_{f}\right)^{r-1}=\left(-e\left(v_{f}\right)\right)^{r-1} \cdot m_{1}$. But $m_{1}=\beta(M)$ and $\beta(M) \cdot \beta\left(v_{f}\right)=\beta\left(\mathbb{R}^{n}\right)=1$, so we end up with

$$
m_{r}=\left(-e\left(v_{f}\right)\right)^{r-1} \cdot \beta(M)^{r} .
$$

Now we can state and prove the main result of this section.

Theorem 2.1 Let $g_{i}: M_{i}^{n_{i}} \rightarrow \mathbb{R}^{n_{i}+k_{i}} ;(i=1,2)$ be generic immersions. Then

$$
\widetilde{M}_{r}\left(g_{1} \times g_{2}\right) \sim(-1)^{r-1} \widetilde{M}_{r}\left(g_{1}\right) \times \widetilde{M}_{r}\left(g_{2}\right)
$$

where stands for "unoriented-cobordant".

If both manifolds $M_{i}$ are oriented and both codimensions $k_{i}$ are even, then the two sides of (2) are oriented cobordant.

Proof We will only consider the oriented case. The unoriented version is proved exactly the same way, except that there is no need to study Pontrjagin classes.

Let $f=g_{1} \times g_{2}$. Then

$$
\begin{aligned}
m_{r}(f) & =\left(-e\left(v_{f}\right)\right)^{r-1} \cdot \beta\left(\left(M_{1} \times M_{2}\right)\right)^{r} \\
& =\left(-e\left(v_{g_{1}} \times v_{g_{2}}\right)\right)^{r-1} \cdot \beta\left(T M_{1} \times T M_{2}\right)^{r} \\
& =(-1)^{r-1}\left(\left(-e\left(v_{g_{1}}\right)^{r-1} \cdot \beta\left(M_{1}\right)^{r}\right) \times\left(\left(-e\left(v_{g_{2}}\right)^{r-1} \cdot \beta\left(M_{2}\right)^{r}\right)\right.\right. \\
& =(-1)^{r-1} m_{r}\left(g_{1}\right) \times m_{r}\left(g_{2}\right) .
\end{aligned}
$$


The following equations are easily checked.

$$
\begin{aligned}
\left\langle\beta\left(\widetilde{M}_{r}(f)\right),\left[\widetilde{M}_{r}(f)\right]\right\rangle & =\left\langle m_{r}(f),\left[M_{1} \times M_{2}\right]\right\rangle \\
& =\left\langle(-1)^{r-1} m_{r}\left(g_{1}\right) \times m_{r}\left(g_{2}\right),\left[M_{1} \times M_{2}\right]\right\rangle \\
& =(-1)^{r-1}\left\langle\beta ( \widetilde { M } _ { r } ( g _ { 1 } ) , [ \widetilde { M } _ { r } ( g _ { 1 } ) ] \rangle \cdot \left\langle\beta\left(\widetilde{M}_{r}\left(g_{2}\right),\left[\widetilde{M}_{r}\left(g_{2}\right)\right]\right\rangle\right.\right. \\
& =(-1)^{r-1}\left\langle\beta\left(\left(\widetilde{M}_{r}\left(g_{1}\right) \times \widetilde{M}_{r}\left(g_{2}\right)\right)\right),\left[\widetilde{M}_{r}\left(g_{1}\right) \times \widetilde{M}_{r}\left(g_{2}\right)\right]\right\rangle .
\end{aligned}
$$

We have obtained equality of two formal power series, so the corresponding coefficients must be equal on the two sides. As the coefficients are the Pontrjagin numbers of the manifolds involved, we get that the Pontrjagin numbers of the two manifolds are all equal.

To finish the proof we have to repeat the whole argument using an analogous class instead of $\beta$, namely

$$
\beta^{\prime}(\xi)=\prod_{i=1}^{\infty}\left(1+w_{1}(\xi) t_{i}^{1}+w_{2}(\xi) t_{i}^{2}+\cdots\right) \in H^{*}\left(B, \mathbb{Z}_{2}\right) \llbracket t_{1}, t_{2}, \ldots \rrbracket .
$$

It is obvious that all the above hold for $\beta^{\prime}$ as well. Thus not only the Pontrjagin numbers, but also the Stiefel-Whitney numbers of the two manifolds are equal. Since the oriented cobordism class is determined by these numbers, the claim of the theorem follows.

This result will no longer hold if we consider a general target space $N$. However the Pontrjagin and Stiefel-Whitney numbers of the multiple-point manifolds of $g_{1} \times g_{2}$ are still expressible in terms of $g_{1}, g_{2}$ and their multiple-point manifolds. This expression is particularly simple for the double-point set.

First we need a simple result about the embedded manifold representing a vector bundle's Euler class. Let $\xi \rightarrow B$ be a vector bundle over a manifold $B$. Let $s: B \rightarrow \xi$ be a section transverse to the 0 -section. Let us denote by $\Delta_{\xi}$ the submanifold in $B$ that is the inverse image of the 0 -section by $s$, and let $\delta_{\xi}: \Delta_{\xi} \rightarrow B$ denote the inclusion.

Lemma 2.2 $\left\langle\beta\left(\Delta_{\xi}\right),\left[\Delta_{\xi}\right]\right\rangle=\langle\beta(B) \cdot(e(\xi) / \beta(\xi)),[B]\rangle$.

Proof It suffices to show that

$$
\delta_{\xi !}\left(\beta\left(\Delta_{\xi}\right)\right)=\beta(B) \cdot \frac{e(\xi)}{\beta(\xi)} .
$$


By the construction of $\Delta_{\xi}$ we have the following pullback diagram:

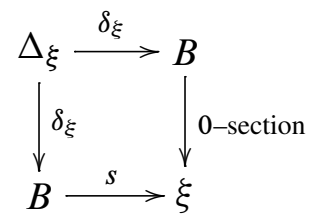

Hence the normal bundle of $\delta_{\xi}$ is just the pullback of the normal-bundle of the 0 -section. This latter is just $\xi$. Thus we have

$$
T \Delta_{\xi} \oplus \delta_{\xi}^{*} \xi=\delta_{\xi}^{*} T B
$$

which in turn implies that

$$
\beta\left(\Delta_{\xi}\right)=\delta_{\xi}^{*}\left(\frac{\beta(B)}{\beta(\xi)}\right) .
$$

Applying the pushforward to this equation gives the conclusion of the lemma, since $f_{!}\left(f^{*} x\right)=f_{!}(1) \cdot x$ is well known and obviously $\delta_{\xi !}(1)=e(\xi)$.

Theorem 2.3 Let $g_{i}: M_{i}^{n_{i}} \rightarrow N_{i}^{n_{i}+k_{i}}, i=1,2$, be generic immersions. Then

$$
\widetilde{M}_{2}\left(g_{1} \times g_{2}\right) \sim \widetilde{M}_{2}\left(g_{1}\right) \times \widetilde{M}_{2}\left(g_{2}\right)+\widetilde{M}_{2}\left(g_{1}\right) \times \Delta_{v_{g_{2}}}+\Delta_{v_{g_{1}}} \times \widetilde{M}_{2}\left(g_{2}\right)
$$

where $\sim$ stands for "unoriented-cobordant". (Recall that $v_{g_{i}}$ is the normal bundle of $g_{i}$ and $\Delta_{g_{i}}$ is the zero set of a generic section of $v_{g_{i}}$.) If the $M_{i}$ are oriented and the $k_{i}$ are even, then the same is true up to oriented cobordism.

Proof We proceed in a similar manner as in the previous theorem. Let us put $f=$ $g_{1} \times g_{2}$ and $M=M_{1} \times M_{2}$ again. Then using (1) we get

$$
\begin{aligned}
& \beta\left(v_{f}\right) \cdot m_{2}(f)=f^{*} \beta(N)-e\left(v_{f}\right) \cdot \beta(M)=g_{1}^{*} \beta\left(N_{1}\right) \times g_{2}^{*} \beta\left(N_{2}\right)-e\left(v_{f}\right) \cdot \beta(M) \\
& =\left(\beta\left(v_{g_{1}}\right) m_{2}\left(g_{1}\right)+e\left(v_{g_{1}}\right) \cdot \beta\left(M_{1}\right)\right) \times\left(\beta\left(v_{g_{2}}\right) m_{2}\left(g_{2}\right)+e\left(v_{g_{2}}\right) \cdot \beta\left(M_{2}\right)\right)-e\left(v_{f}\right) \cdot \beta(M) \\
& =\beta\left(v_{f}\right) \cdot\left(m_{2}\left(g_{1}\right) \times m_{2}\left(g_{2}\right)+m_{2}\left(g_{1}\right) \times \beta\left(M_{2}\right) \frac{e_{v_{g_{2}}}}{\beta\left(v_{g_{2}}\right)}+\beta\left(M_{1}\right) \frac{e_{v_{1}}}{\beta\left(v_{g_{1}}\right)} \times m_{2}\left(g_{2}\right)\right) .
\end{aligned}
$$

Now we can divide by $\beta\left(v_{f}\right)$ as it is an invertible element. We evaluate both sides on $[M]=\left[M_{1}\right] \times\left[M_{2}\right]$. Finally we have to apply the previous lemma to get that all the corresponding characteristic numbers are equal for the two manifolds in question. As before, we can repeat the argument for Stiefel-Whitney numbers in $\mathbb{Z}_{2}$ coefficients and Pontrjagin numbers in $\mathbb{Q}$ coefficients, so we get both parts of the theorem at the same time. 
Remark (1) It is possible to carry out similar calculations for triple points or points of higher (say $r$ ) multiplicity. But the number of terms involved in these formulas grow exponentially with $r$ and the authors did not manage to find a nice way to write them down, not even recursively.

(2) It would be possible to obtain similar formulas not only for the cobordism classes of the underlying multiple-point manifolds, but for the cobordism classes of the immersions $\phi_{r}$ themselves. To do this one would need to consider the characteristic numbers of these immersions instead of the characteristic numbers of the manifolds. These calculations are more or less the same as the ones described here, but they are harder to keep track of.

(3) It seems that the same results could be obtained using techniques of Eccles and Grant from [4].

(4) We would like to point out that Theorem 2.3 is a nontrivial generalization of the oriented case of Theorem A in Byun and Yi [3], which considers the case of $n=k$.

\section{Ring structure of Morin maps}

Given a smooth map $f: M \rightarrow N$ where $\operatorname{dim} M \leq \operatorname{dim} N$, a point $x \in M$ is said to be a $\Sigma^{i}$ point if the corank (ie the dimension of the kernel) of $d f_{x}: T_{x} M \rightarrow T_{f(x)} N$ is $i$. The set of such points is denoted by $\Sigma^{i}(f)$. If $i_{1} \geq i_{2}$ then we can define $\Sigma^{i_{1}, i_{2}}(f)=\Sigma^{i_{2}}\left(\left.f\right|_{\Sigma^{i_{1}}(f)}\right)$. This method can be continued recursively to give the definition of $\Sigma^{\left(i_{1}, i_{2}, \ldots, i_{r}\right)}$ points, where $i_{1} \geq i_{2} \geq \cdots \geq i_{r}$. This classification of singular points is called the Thom-Boardman type. For details see eg Arnol'd et al [1].

A generic smooth map $f: M \rightarrow N$ is called a Morin map if it has no $\Sigma^{2}$ points. The singularities of such maps are classified by their Thom-Boardman type, which can only be

$$
\Sigma \overbrace{(1,1, \ldots, 1)}^{r}=\Sigma^{1^{1}}
$$

for some $r \geq 0$. (In the notation of [1] this is $A_{r}$.)

Cobordism of Morin maps is defined as usual: two Morin maps $f: M_{1}^{n} \rightarrow N^{n+k}$ and $g: M_{2}^{n} \rightarrow N^{n+k}$ are said to be cobordant if there is a Morin map $H: W^{n+1} \rightarrow$ $N^{n+k} \times[0,1]$ such that $\partial W=M_{1} \cup M_{2}$ and $\left.H\right|_{M_{1} \times[0, \varepsilon)}=f \times \operatorname{id}_{[0, \varepsilon)},\left.H\right|_{M_{2} \times(1-\varepsilon, 1]}=$ $g \times \operatorname{id}_{(1-\varepsilon, 1]}$ for a sufficiently small $\varepsilon$.

Let us consider the set of cobordism classes of all Morin maps to Euclidean spaces (for all nonnegative dimensions and all positive codimensions). This set is a bigraded 
commutative group with addition induced by the disjoint union of maps. We can take tensor product with $\mathbb{Q}$ to obtain the rational cobordism group of Morin maps whose elements will be referred to as rational cobordism classes. In this section we endow this rational cobordism group with a bigraded ring structure. Further we will show that the singularities can be used to define ring homomorphisms into $\Omega_{*} \otimes \mathbb{Q}$, where $\Omega_{*}$ is the oriented cobordism ring of manifolds.

The main tool in constructing the multiplication will be the so-called "prim maps", while the ring homomorphisms will be derived from the results of the previous section.

\subsection{Prim maps}

Definition 3.1 A generic map $f: M \rightarrow N$ is called prim (projected immersion) if it can be lifted to a generic immersion, $\tilde{f}: M \rightarrow N \times \mathbb{R}$, and this lifting is fixed up to regular homotopy. (This is equivalent to saying that the kernel bundle of $d f$ is trivialised.) We will always denote the lifting by a tilde.

Cobordism of prim maps can be defined in a natural way (the cobordism itself should be a prim map into $N \times[0,1]$ ), and disjoint union induces a group operation on the cobordism classes. The class of a prim map $f$ will be denoted by $[f]$. (For details see eg Szúcs [7].)

Clearly a prim map is necessarily a Morin map. Prim maps provide a good link between immersions and Morin maps. We shall first define multiplication of prim maps (using their liftings to immersions) and then show how to extend it to multiplication of Morin maps (using results of [9]). We will only work with prim maps whose target space is Euclidean.

Let us denote $l_{0}: \mathrm{pt} \hookrightarrow \mathbb{R}$ the inclusion of a point into the line.

Lemma 3.2 (a) Any two generic hyperplane projections of an immersion represent the same prim cobordism class.

(b) Hyperplane projections of cobordant immersions represent the same prim cobordism class.

Proof (a) Instead of taking two projections of the same immersion we can take projection to the same hyperplane of two immersions which differ only by a rotation. This rotation can be realized by a regular homotopy. Regular homotopy is a special case of cobordism, hence a) will follow from $b$ ).

(b) A generic hyperplane projection of the cobordism connecting the two immersions gives a prim cobordism of the prim maps obtained on the boundaries. 
Definition 3.3 For two prim maps $f_{i}: M_{i} \rightarrow \mathbb{R}^{n_{i}}, i=1,2$, consider the product map

$$
g=f_{1} \times f_{2} \times l_{0}: M_{1} \times M_{2} \rightarrow \mathbb{R}^{n_{1}+n_{2}} \times \mathbb{R} .
$$

The map $g$ might not yet be prim, but we can turn it into such by a small perturbation. Take liftings $\widetilde{f}_{1}$ and $\widetilde{f}_{2}$ that are sufficiently close to $f_{1} \times l_{0}$ and $f_{2} \times l_{0}$. Now $\tilde{f}_{1} \times \widetilde{f}_{2}: M_{1} \times M_{2} \rightarrow \mathbb{R}^{n_{1}+n_{2}} \times \mathbb{R}^{2}$ is a nongeneric immersion. Let us take a sufficiently small perturbation of this product so that it becomes a generic immersion. Finally take a generic projection of this immersion to a hyperplane "close" to $\mathbb{R}^{n_{1}+n_{2}} \times \mathbb{R}$, where the last $\mathbb{R}$ factor is the diagonal in $\mathbb{R}^{2}$. We obviously get a prim map $g^{\prime}$ that can be arbitrarily close to $g$. Let us denote $g^{\prime}=f_{1} * f_{2}$ and let us define the multiplication on prim cobordism classes as follows: $\left[f_{1}\right] *\left[f_{2}\right]=\left[f_{1} * f_{2}\right]$.

Theorem 3.4 The above definition is correct, that is the cobordism class $\left[f_{1} * f_{2}\right]$ is independent of the choice of $f_{1}$ and $f_{2}$ within their cobordism class and of any other choices made in the definition. The multiplication defined in this way together with the addition being the disjoint union gives rise to a ring structure.

Proof The liftings are given up to regular homotopy. Also the perturbation of $\tilde{f}_{1} \times \tilde{f}_{2}$ is unique up to regular homotopy. Thus Lemma 3.2 implies that the resulting prim map is independent of these choices.

Now suppose $\left[f_{1}\right]=\left[g_{1}\right]$. Then there is a prim cobordism $H$ joining $f_{1}$ and $g_{1}$. We can take its lifting $\widetilde{H}$ which is an immersed cobordism between $\widetilde{f}_{1}$ and $\widetilde{g_{1}}$, and so $\widetilde{f}_{1} \times \widetilde{f}_{2}$ and $\widetilde{g}_{1} \times \widetilde{f}_{2}$ are regularly homotopic via $\tilde{H} \times \widetilde{f}_{2}$. So their projections are prim cobordant, and this is what we wanted to prove. (The definition is symmetric so the other factor can be handled the same way.)

The last claim only requires checking distributivity, which is obvious.

\subsection{Morin maps}

In this section we only consider maps between oriented manifolds. Let us denote the group of cobordism classes of oriented Morin maps $f: M^{n} \rightarrow \mathbb{R}^{n+k}$ by $\operatorname{Cob}_{\Sigma^{1}}(n, k)$ and the cobordism classes of oriented prim maps $f: M^{n} \rightarrow \mathbb{R}^{n+k}$ by $\operatorname{Prim}(n, k)$. As a prim map is automatically Morin and prim cobordant maps are Morin cobordant as well, we have a natural forgetful map $F: \operatorname{Prim}(n, k) \rightarrow \operatorname{Cob}_{\Sigma^{1}}(n, k)$, that induces a $\operatorname{map} F_{\mathbb{Q}}: \operatorname{Prim}(n, k) \otimes \mathbb{Q} \rightarrow \operatorname{Cob}_{\Sigma^{1}}(n, k) \otimes \mathbb{Q}$. The following key result is proved in [9] as Remark 103.

Lemma 3.5 The map $F_{\mathbb{Q}}$ is epimorphic. 
This lemma says that every Morin map has a nonzero multiple that is Morin-cobordant to a prim map. Using this result and the construction in the previous section we can now define a multiplication on $\left(\bigoplus_{n, k} \operatorname{Cob}_{\Sigma^{1}}(n, k)\right) \otimes \mathbb{Q}$.

Definition 3.6 Let us take two Morin maps $g_{i}: M_{i}^{n_{i}} \rightarrow \mathbb{R}^{n_{i}+k_{i}}$. By Lemma 3.5 we can find prim maps $f_{1}$ and $f_{2}$ that are rationally Morin cobordant to $g_{1}$ and $g_{2}$. Let us define $\left[g_{1}\right] *\left[g_{2}\right]:=F_{\mathbb{Q}}\left(\left[f_{1} * f_{2}\right]\right)$, where $[f]$ denotes the rational Morin cobordism class of the Morin map $f$.

Theorem 3.7 The above definition is correct, that is $\left[g_{1}\right] *\left[g_{2}\right]$ is independent of the choices made. The multiplication defined this way gives rise to a ring structure on $\left(\bigoplus_{n, k} \operatorname{Cob}_{\Sigma^{1}}(n, k)\right) \otimes \mathbb{Q}$.

Proof There is only one thing left that needs to be checked: if $f_{1}$ and $f_{1}^{\prime}$ are Morin cobordant prim-representatives of $g_{1}$, then $f_{1} * f_{2}$ is Morin cobordant to $f_{1}^{\prime} * f_{2}$. Let us take the Morin cobordism $H$ connecting $f_{1}$ and $f_{1}^{\prime}$. Then $H \times\left(f_{2} \times l_{0}\right)$ is still a Morin cobordism after a sufficiently small perturbation, since the factor $f_{2} \times l_{0}$ can be perturbed to an immersion. This Morin cobordism connects exactly the two desired maps.

Definition 3.8 Let Mor $_{\mathbb{Q}}$ denote the group $\bigoplus_{n, k} \operatorname{Cob}_{\Sigma^{1}}(n, k) \otimes \mathbb{Q}$ with this ring structure. Mor $_{\mathbb{Q}}$ is a bigraded ring, the two grades being $n$ and $k+1$. Note that this implies that the direct sum $\bigoplus_{k \text { odd, } n} \operatorname{Cob}_{\Sigma^{1}}(n, k) \otimes \mathbb{Q}$ is a subring of $\operatorname{Mor}_{\mathbb{Q}}$.

\section{Computing Mor $_{\mathbb{Q}}$}

In this section we show that the rational cobordism class of an oriented Morin map is actually determined by those of its singular strata. Then we will show that the singular strata are ring homomorphisms from Mor $_{\mathbb{Q}}$. This provides a complete computation of the ring $\operatorname{Mor}_{\mathbb{Q}}$.

\subsection{The structure of $\operatorname{Cob}_{\Sigma}{ }^{1}(n, k)$}

Let $f: M^{n} \rightarrow \mathbb{R}^{n+k}$ be a generic oriented Morin map of codimension $k$. To such a map we can associate the subset $\Sigma^{1 r}(f) \subset M^{n}$ introduced at the beginning of Section 3. This subset is actually a submanifold whose codimension is $r \cdot(k+1)$. The cobordism class of this submanifold is invariant under a Morin cobordism of $f$, since the $\Sigma^{1_{r}}$ points of the cobordism of $f$ give a cobordism between the $\Sigma^{1^{r}}$ points of $f$. 
If we tensor with $\mathbb{Q}$ then this submanifold becomes an element in $\Omega_{*} \otimes \mathbb{Q}$. Thus by abuse of notation we get maps

$$
\Sigma^{1_{r}}: \operatorname{Cob}_{\Sigma^{1}}(n, k) \otimes \mathbb{Q} \rightarrow \Omega_{*} \otimes \mathbb{Q}, \quad[f] \mapsto\left[\Sigma^{1_{r}}(f)\right],
$$

whose image we denote by $\operatorname{Im}\left(\Sigma^{1 r}\right)$. Let $\operatorname{Imm}^{\mathrm{SO}}(n, k)$ denote the oriented cobordism group of immersions of $n$-dimensional oriented manifolds to $\mathbb{R}^{n+k}$. Our goal in this section is to prove the following theorem:

Theorem $4.1 \quad$ (i) If $k=2 l$ then $\operatorname{Cob}_{\Sigma^{1}}(n, k) \otimes \mathbb{Q}=\operatorname{Imm}^{\mathrm{SO}}(n, k+1) \otimes \mathbb{Q}$ and $\operatorname{Im}\left(\Sigma^{1^{r}}\right)=0$ for any $r \geq 1$.

(ii) If $k=2 l+1$ then

$$
\operatorname{Cob}_{\Sigma^{1}}(n, k) \otimes \mathbb{Q}=\bigoplus_{r \geq 0} \operatorname{Im}\left(\Sigma^{1_{r}}\right)=\bigoplus_{i \geq 0} \operatorname{Im}\left(\Sigma^{1_{2 i}}\right)
$$

where $\operatorname{Im}\left(\Sigma^{1_{2 i+1}}\right)=0$ and $\operatorname{Im}\left(\Sigma^{1_{2 i}}\right)=\left\{[L] \in \Omega_{n-2 i(k+1)} \otimes \mathbb{Q}: p_{I}[L]=0\right.$ for any Pontrjagin monomial $p_{I}$ which has a factor $p_{j}$ with index $\left.j \geq l\right\}$

Proof Part (i) is stated explicitly in [9] in Section 14/A.

To show part (ii) we need some preparations. For any stable singularity type $\eta$ there is a bundle $\widetilde{\xi}_{\eta}$ that plays the role of the universal normal bundle for this singularity type. This means the following: Whenever for a map $f: M \rightarrow N$ one of its most complicated singularities is $\eta$ then the $\eta$-points of $f$ form a submanifold of $M$. The restriction of $f$ to this submanifold is an immersion to $N$. The normal bundle of this immersion is induced from $\widetilde{\xi}_{\eta}$. (See Rimányi and Szúcs [5] for details.)

Let us write $\widetilde{\xi}_{r}=\widetilde{\xi}_{\Sigma^{1}{ }^{1}}$ for short. Let $\operatorname{Imm} \widetilde{\xi}_{r}(n-r(k+1), r(k+1)+k)$ denote the cobordism group of oriented immersions $f: M^{n-r(k+1)} \rightarrow \mathbb{R}^{n+k}$ whose normal bundles are induced from $\tilde{\xi}_{r}$.

We need two results from [9] which we state here in a lemma.

Lemma 4.2 Let $k \geq 1$ be odd. Then

$$
\begin{aligned}
\operatorname{Cob}_{\Sigma^{1}}(n, k) \otimes \mathbb{Q} & \cong \bigoplus_{i=0}^{\infty} \operatorname{Imm}^{\tilde{\xi}_{2 i}}(n-2 i(k+1), 2 i(k+1)+k) \otimes \mathbb{Q}, \\
H_{n+k}\left(T \widetilde{\xi}_{2 i} ; \mathbb{Q}\right) & =H_{n-2 i(k+1)}(B \operatorname{SO}(k) ; \mathbb{Q}) .
\end{aligned}
$$

Remark The above isomorphism (3) is given roughly speaking by the direct sum of the maps $\Sigma^{2 i}$, for all nonnegative integer values of $i$. 
More precisely for a map $f$ that represents the cobordism class $[f] \in \operatorname{Cob}_{\Sigma^{1}}(n, k)$ the isomorphism of (3) is defined by successively removing the most singular points from $f$ by cobordism. (Note that if $r(k+1)>n$ than $f$ has no $\Sigma^{1} r$ singular points.) In each step we take the submanifold consisting of the most singular points of the map. This submanifold is immersed into $\mathbb{R}^{n+k}$ - by the restriction of the map to it - and has the normal $\widetilde{\xi}_{s}$-structure (if this is the submanifold of $\Sigma^{1_{s}}$ points), and thus gives the $s$-th coordinate of the image of $[f]$ on the right hand side. (It turns out that for $s$ odd the cobordism class of this immersion is always trivial rationally, that is why we have on the right hand side summands only for $s$ even.)

Proof of Lemma 4.2 Equation (3) is an immediate consequence of [9, Example 118]. For Equation (4) we cite from [9] that the bundle $\widetilde{\xi}_{\eta}$ has a counterpart denoted by $\xi_{\eta}$ which is the universal normal bundle of the $\eta$-points of a map in the source manifold. The two bundles $\xi_{\eta}$ and $\widetilde{\xi}_{\eta}$ have the same base space $B G_{\eta}$ where $G_{\eta}$ is the maximal compact subgroup of the symmetry group of the singularity $\eta$. This implies that the homologies of $T \widetilde{\xi}_{\eta}$ and $T \xi_{\eta}$ are the same up to a dimension shift equal to $\operatorname{rank} \widetilde{\xi}_{\eta}-\operatorname{rank} \xi_{\eta}=k$, that is, $H_{n+k}\left(T \widetilde{\xi}_{r} ; \mathbb{Q}\right)=H_{n}\left(T \xi_{r} ; \mathbb{Q}\right)$. Lemma $102 / \mathrm{b}$ in [9] implies that for even $r$ we have $H_{n}\left(T \xi_{r} ; \mathbb{Q}\right)=H_{n-r(k+1)}(B \operatorname{SO}(k) ; \mathbb{Q})$. The statement follows.

Now we can return to the proof of Theorem 4.1 which will follow fairly easily from (3) of Lemma 4.2. Let us introduce the notation $a=n-r(k+1)$ and $b=r(k+1)+k=$ $n+k-a$ for convenience. Let us consider the sequence of forgetful maps

$$
\operatorname{Imm}^{\tilde{\xi}_{r}}(a, b) \stackrel{\widetilde{\alpha}}{\rightarrow} \operatorname{Imm}^{\mathrm{SO}}(a, b) \stackrel{\widetilde{\beta}}{\rightarrow} \Omega_{a},
$$

where we first forget about the extra structure on the normal bundle, and then forget about the immersion and just take the underlying source manifold. On the level of Thom spaces this corresponds to the standard maps

$$
T \tilde{\xi}_{r} \rightarrow T \gamma_{b}^{\mathrm{SO}} \rightarrow T \gamma^{\mathrm{SO}}
$$

and on the level of base spaces to

$$
B \mathrm{SO}(k) \stackrel{\alpha}{\rightarrow} B \mathrm{SO}(b) \stackrel{\beta}{\rightarrow} B \mathrm{SO} .
$$

The map $\alpha$ induces $\tilde{\xi}_{r}$ from $\gamma_{b}^{\text {SO }}$ and $\beta$ is just the standard inclusion map.

Using the well-known Pontrjagin-Thom construction and (4) of Lemma 4.2 we get that

$$
\operatorname{Imm}^{\tilde{\xi}_{r}}(a, b) \otimes \mathbb{Q} \cong \pi_{n+k}^{S}\left(T \tilde{\xi}_{r}\right) \otimes \mathbb{Q} \cong H_{n+k}\left(T \tilde{\xi}_{r} ; \mathbb{Q}\right)=H_{a}(B \operatorname{SO}(k) ; \mathbb{Q}) \text {. }
$$


Similarly we have

$$
\operatorname{Imm}^{\mathrm{SO}}(a, b) \otimes \mathbb{Q} \cong \pi_{n+k}^{S}\left(T \gamma_{b}^{\mathrm{SO}}\right) \otimes \mathbb{Q} \cong H_{n+k}\left(T \gamma_{b}^{\mathrm{SO}} ; \mathbb{Q}\right)=H_{a}(B \operatorname{SO}(b) ; \mathbb{Q}),
$$

and $\Omega_{a} \otimes \mathbb{Q} \cong H_{a}(B S O ; \mathbb{Q})$. In this context the forgetful map in (5) becomes

$$
H_{a}(B S O(k) ; \mathbb{Q}) \stackrel{\alpha_{*}}{\rightarrow} H_{a}(B S O(b) ; \mathbb{Q}) \stackrel{\beta_{*}}{\rightarrow} H_{a}(B S O ; \mathbb{Q}) .
$$

We want to prove that this map is injective, and compute its image. Since we work with rational coefficients it is enough to show that the dual map

$$
H^{a}(B \mathrm{SO}(k) ; Q) \stackrel{\alpha^{*}}{\leftarrow} H^{a}(B \mathrm{SO}(b) ; \mathbb{Q}) \stackrel{\beta^{*}}{\leftarrow} H^{a}(B \mathrm{SO} ; \mathbb{Q})
$$

is surjective, and compute its kernel. $H^{*}(B S O ; \mathbb{Q})=\mathbb{Q}\left[p_{1}, p_{2}, \ldots\right]$ and since $k$ is odd $H^{*}(B \operatorname{SO}(k) ; \mathbb{Q})=\mathbb{Q}\left[p_{1}, p_{2}, \ldots, p_{(k-1) / 2}\right]$. The induced homomorphism $\alpha^{*} \beta^{*}$ takes the total Pontrjagin class $p=1+p_{1}+p_{2}+\cdots$ to the total Pontrjagin class of $\widetilde{\xi}_{r}$ preserving the grading. It is known (see [8, Lemma 2]) that $p\left(\widetilde{\xi}_{r}\right)=p\left(\gamma_{k}^{\mathrm{SO}}\right)^{r}=$ $\left(1+p_{1}+p_{2}+\cdots+p_{(k-1) / 2}\right)^{r} \in H^{*}(B \mathrm{SO}(k) ; \mathbb{Q})$. Now easy computation shows that indeed every $p_{i} \in H^{*}(B \operatorname{SO}(k) ; \mathbb{Q})$ is in the image of $\alpha^{*} \beta^{*}$ so the map is surjective. On the other hand the kernel is generated exactly by those Pontrjagin monomials in which there is at least one factor with index larger than $(k-1) / 2$. Thus the image of $\widetilde{\beta} \widetilde{\alpha}: \operatorname{Imm}^{\tilde{\xi}_{r}}(a, b) \rightarrow \Omega_{a}$ is generated by the cobordism classes of exactly those manifolds $L$ for which $p_{I}[L]=0$ for any Pontrjagin monomial $p_{I}$ which has a factor $p_{j}$ with index $j \geq(k-1) / 2$.

To finish the proof of the theorem we just have to observe that $\Sigma^{1_{r}}$ is the composition of the projection in the splitting (3) and the forgetful map in (5): $\operatorname{Cob}_{\Sigma^{1}}(n, k) \otimes \mathbb{Q} \rightarrow$ $\operatorname{Imm}^{\widetilde{\xi}_{r}}(n-r(k+1), r(k+1)+k) \otimes \mathbb{Q} \rightarrow \Omega_{n-r(k+1)} \otimes \mathbb{Q}$.

\subsection{Ring homomorphisms}

We can consider $\Sigma^{1_{r}}$ as a map from Mor $\mathbb{Q}_{\mathbb{Q}}$ to the rational oriented cobordism ring:

$$
\Sigma^{1^{r}}: \bigoplus_{k, n} \operatorname{Cob}_{\Sigma^{1}}(n, k) \otimes \mathbb{Q} \rightarrow \Omega_{*} \otimes \mathbb{Q}
$$

Theorem 4.3 The map $\Sigma^{1_{r}}$ is a ring homomorphism. In other words

$$
\Sigma^{1_{r}}(f * g) \sim \Sigma^{1_{r}}(f) \times \Sigma^{1_{r}}(g)
$$

holds for any two Morin maps $f, g$ to Euclidean spaces where $\sim$ now stands for rationally cobordant (in the oriented sense). 
Proof The only case that requires proof is when $k$ is odd and $r>0$ is even, since otherwise $\Sigma^{1^{r}}(f)$ is always 0 and for $r=0$ the statement is obvious. (Note that $\Sigma^{0}(f)=\Sigma^{1_{0}}(f)$ is the rational cobordism class of the source manifold of $f$.) We will proceed along the lines explained earlier, that is we will use prim maps as a link between Morin maps and immersions. Then the multiplicative properties of multiple points of immersions will provide the result.

Let us first consider prim maps. The same argument as above gives a map

$$
\Sigma_{\text {Prim }}^{1 r}:\left(\bigoplus_{k \text { odd }, n} \operatorname{Prim}(n, k)\right) \otimes \mathbb{Q} \rightarrow \Omega_{*} \otimes \mathbb{Q}
$$

where $\Sigma_{\text {Prim }}^{1_{r}}=\Sigma^{1_{r}} \circ F_{\mathbb{Q}}$.

Given an immersion $f: M^{n} \rightarrow \mathbb{R}^{n+k+1}$, let us denote by $\pi(f)$ its generic projection to a hyperplane. This map is a prim map whose prim cobordism class is well defined and depends only on the cobordism class of the immersion $f$ according to Lemma 3.2. The direct sum $\bigoplus_{k \text { odd, } n} \operatorname{Imm}^{\mathrm{SO}}(n, k+1)$ has a natural ring structure with multiplication being the direct product. It is clear from the definitions that the map

$$
\pi: \bigoplus_{k \text { odd }, n} \operatorname{Imm}^{\mathrm{SO}}(n, k+1) \rightarrow \bigoplus_{k \text { odd }, n} \operatorname{Prim}(n, k), \quad[f] \mapsto[\pi(f)]
$$

is a ring homomorphism with respect to the direct product on the left, and $*$-product on the right. The same remains true after forming the tensor product with $\mathbb{Q}$.

In Theorem 2.1 we have shown that

$$
\widetilde{M}_{r+1}: \bigoplus_{k \text { odd }, n} \operatorname{Imm}^{\mathrm{SO}}(n, k+1) \rightarrow \Omega_{*}
$$

is a ring homomorphism, and obviously the same is true after forming the tensor product with $\mathbb{Q}$.

To finish the proof we have to recall a result from [8] which in our notation reads as:

Theorem 4.4 $\widetilde{M}_{r+1} \otimes \mathrm{id}_{\mathbb{Q}}=\Sigma_{\text {Prim }}^{1_{r}} \circ\left(\pi \otimes \mathrm{id}_{\mathbb{Q}}\right)$ ie the rational cobordism class of the manifold of $r+1$-tuple points of an immersion $f: M^{n} \rightarrow R^{n+k+1}$ coincides with that of the manifold of $\Sigma^{1_{r}}$ (or $A_{r}$ ) points of its hyperplane projection. 
Thus the following diagram is commutative.

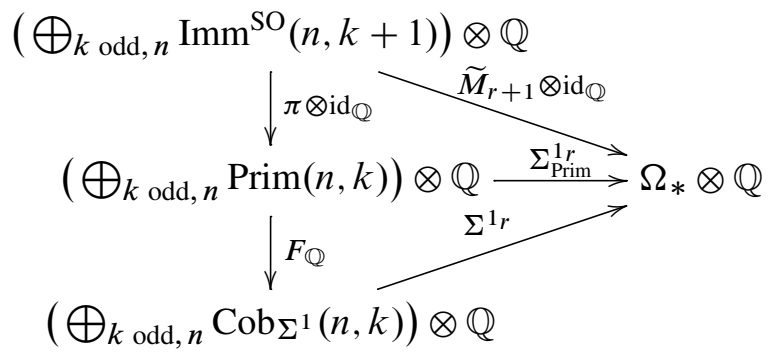

The vertical maps are ring epimorphisms and $\widetilde{M}_{r+1}$ is a ring homomorphism. This implies that $\Sigma_{\text {Prim }}^{1_{r}}$ and $\Sigma^{1_{r}}$ are ring homomorphisms too.

We can summarize our results as follows. The ring $\operatorname{Mor}_{\mathbb{Q}}$ is the direct sum of clearly identified subgroups of $\Omega_{*} \otimes \mathbb{Q}$ as stated in Theorem 4.1. An element in Mor $\mathbb{Q}$ is completely determined by its grading and its collection of $\Sigma^{1_{r}}$ strata. Then Theorem 4.3 shows that the multiplication in Mor $_{\mathbb{Q}}$ corresponds to the direct product of cobordism classes representing the singular strata. The manifolds corresponding to the various $\Sigma^{1_{r}}$ strata multiply independently of each other. Thus the ring Mor $\mathbb{Q}_{\mathbb{Q}}$ is completely computed.

\section{Singular strata of direct products}

Our goal in this final section is to show that the cohomology class represented by the submanifold formed by the closure of the set of certain singular points of a direct product $f \times g$ depends only on those of $f, g$ and some maps closely related to them. Before formulating the theorems, we have to introduce some notation.

Definition 5.1 For $j>0$ let $q_{j}: * \rightarrow S^{j}$ denote the inclusion of a point into $S^{j}$ and let $q_{(-j)}: S^{j} \rightarrow *$ be the map that takes the sphere to a point. Now for any integer $j \neq 0$ we define $f_{j}^{\prime}=f \times q_{j}$, take $f_{j}$ to be a generic perturbation of $f_{j}^{\prime}$, and set $f_{0}=f$.

Finally let $\mathrm{id}_{M}^{j}=\mathrm{id}_{M} \times q_{j}: M \rightarrow M \times S^{j}$.

\subsection{The $\Sigma^{1}$ stratum}

In this section we work with $Z_{2}$ coefficients. Let $\Sigma^{1} f=\Sigma^{1}(f)$ denote the closure of the set of all singular points in the source manifold of $f$. (The parenthesis are omitted for easier reading of the formulas below.) The Thom polynomial of this singularity type is $w_{k+1}$ (see Thom [10]). That is, given a map $f: M^{n} \rightarrow N^{n+k}$, the cohomology class Poincaré dual to the homology class represented by $\Sigma^{1} f$ is equal to $w_{k+1}\left(v_{f}\right)$ 
where $v_{f}$ stands for the virtual normal bundle of $f$. This dual cohomology class will be denoted by $\left[\Sigma^{1} f\right]$ for simplicity.

Theorem 5.2 Let $f: M_{1}^{n_{1}} \rightarrow N^{n_{1}+k_{1}}, g: M_{2}^{n_{2}} \rightarrow N_{2}^{n_{2}+k_{2}}$ be two generic maps. Then for a generic perturbation of their product we have

$$
\left[\Sigma^{1}(f \times g)\right]=\sum_{j \geq 1}\left(\left[\Sigma^{1} f_{j-1}\right] \times\left(\mathrm{id}_{M_{2}}^{j}\right)^{*}\left[\Sigma^{1} g_{(-j)}\right]+\left(\mathrm{id}_{M_{1}}^{j}\right)^{*}\left[\Sigma^{1} f_{(-j)}\right] \times\left[\Sigma^{1} g_{j-1}\right]\right) .
$$

Proof As a first step let us notice that since $v_{f \times g}=v_{f} \times v_{g}$ we can write

$$
\begin{aligned}
w_{k_{1}+k_{2}+1}\left(v_{f \times g}\right) & =\sum_{r=0}^{k_{1}+k_{2}+1} w_{r}\left(v_{f}\right) \times w_{k_{1}+k_{2}+1-r}\left(v_{g}\right) \\
& =\sum_{j \geq 1}\left(w_{k_{1}+j}\left(v_{f}\right) \times w_{k_{2}-j+1}\left(v_{g}\right)+w_{k_{1}-j+1}\left(v_{f}\right) \times w_{k_{2}+j}\left(v_{g}\right)\right) .
\end{aligned}
$$

Now we have to take a closer look at $w_{k_{1}+j}\left(v_{f}\right)$. If $j=1$ then this characteristic class just represents $\Sigma^{1} f$. When this is not the case, we have to find an appropriate replacement for $f$ that has the right codimension, whose normal bundle however is stably equivalent to that of $f$. This replacement map is exactly $f_{j-1}$. Indeed, $v_{f_{j-1}}=v_{f} \oplus \varepsilon^{j-1}$ so $w_{k_{1}+j}\left(v_{f}\right)=w_{k_{1}+j}\left(v_{f_{j-1}}\right)$ which in turn is equal to [ $\left.\Sigma^{1} f_{j-1}\right]$ since this map has the right codimension.

The argument is just slightly more complicated in the case of $w_{k_{2}-j+1}\left(v_{g}\right)$. Here first we take the map $g_{(-j)}: M_{2}^{n_{2}} \times S^{j} \rightarrow N_{2}^{n_{2}+k_{2}}$. This has codimension $k_{2}-j$ so $\left[\Sigma^{1} g_{(-j)}\right]=w_{k_{2}-j+1}\left(v_{g_{(-j)}}\right)$. The only problem is that this class lives in the cohomology of $M_{2} \times S^{j}$. This is why we have to pull it back to $M_{2}$ by $\mathrm{id}_{M_{2}}^{j}$. Since the composition of $\mathrm{id}_{M_{2}}^{j}$ and $g_{(-j)}$ is just a perturbation of $g$ and $w\left(v_{q_{j}}\right)=1$ it follows that $\left(\mathrm{id}_{M_{2}}^{j}\right)^{*} w_{k_{2}-j+1}\left(v_{g_{(-j)}}\right)=w_{k_{2}-j+1}\left(v_{g}\right)$.

Putting all these together gives the result of the theorem.

\subsection{The $\Sigma^{2}$ stratum}

A very similar result can be proved about the $\Sigma^{2}$ stratum of oriented maps. For that we need the Thom polynomial of the $\Sigma^{2}$ stratum in the oriented case. This is known (see Ronga [6]) but we shall include a new proof for the sake of completeness. We will work with rational coefficients.

Theorem 5.3 Let $f: M^{n} \rightarrow N^{n+k}$ be a generic map where $k=2 t-2$. Then the rational cohomology class dual to the closure of the set of $\Sigma^{2}$-points of $f$ (for short $\left.\left[\Sigma^{2} f\right]\right)$ equals $p_{t}\left(v_{f}\right)$, where $p_{t} \in H^{4 t}(M ; \mathbb{Q})$ is the $t$-th Pontrjagin class. 
Proof By definition the Thom polynomial tp $\Sigma_{\Sigma^{2}}$ of the $\Sigma^{2}$-stratum is a cohomology class in $H^{4 t}(B S O ; \mathbb{Q})=\mathbb{Q}\left[p_{1}, p_{2}, p_{3}, \ldots\right]$. We want to show that $\operatorname{tp}_{\Sigma^{2}}=p_{t}$. It is enough to show that these two cohomology classes evaluated on any homology class in $H_{4 t}(B S O ; \mathbb{Q})$ are equal.

Lemma 5.4 All homology classes in $H_{4 t}(B S O ; \mathbb{Q})$ can be represented by a normal map, ie by a map $h: L^{4 t} \rightarrow B S O$ of an oriented $4 t$-manifold $L^{4 t}$ corresponding to the stable normal bundle of $L^{4 t}$.

Proof It is enough to consider $B \mathrm{SO}(N),(N \gg 1)$. By the Pontrjagin-Thom construction an embedding $L^{4 t} \hookrightarrow S^{K}$ gives a map $h^{\prime}: S^{K} \rightarrow M \mathrm{SO}(K-4 t)$ such that $L^{4 t}=h^{\prime(-1)}(B \operatorname{SO}(K-4 t))$ and the restriction map $\left.h^{\prime}\right|_{L^{4 t}: L^{4 t} \rightarrow B S O}(K-4 t)$ corresponds to the normal bundle of the embedding $L^{4 t} \hookrightarrow S^{K}$. The homotopy class $\left[h^{\prime}\right] \in \pi_{K}(M \mathrm{SO}(K-4 t))$ is mapped by the composition of the Hurewicz homomorphism and the Thom isomorphism into a homology class $x=h_{*}^{\prime}\left(\left[L^{4 t}\right]\right) \in$ $H_{4 t}(B S O(K-4 t))$. Hence this class $x$ is represented by a normal map. Since the Hurewicz homomorphism in stable dimensions $(K \geq 8 t+2)$ is a rational isomorphism, we obtain the statement of the lemma.

To evaluate a $4 t$-dimensional cohomology class on a $4 t$-dimensional homology class represented by a manifold, one just pulls back the cohomology class to the manifold and evaluates it on the fundamental class.

Now it is enough to prove that for every oriented $M^{4 t}$ the map $v^{*}: H^{4 t}(B S O ; \mathbb{Q}) \rightarrow$ $H^{4 t}(M ; \mathbb{Q})$ induced by the normal mapping $v: M^{4 t} \rightarrow B$ SO takes $p_{t}$ and $\operatorname{tp}_{\Sigma^{2}}$ to the same cohomology class in $H^{4 t}(M ; \mathbb{Q})$. As $v^{*}\left(p_{t}\right)=p_{t}\left(v_{M}\right)$ and $v^{*}\left(\operatorname{tp}_{\Sigma^{2}}\right)$ is the dual of the $\Sigma^{2}$ stratum of a generic map $M^{4 t} \rightarrow \mathbb{R}^{6 t-2}$ we have reduced the problem of finding the Thom polynomial tp $\Sigma_{\Sigma^{2}}$ to the special case of maps $M^{4 t} \rightarrow \mathbb{R}^{6 t-2}$.

If we take an immersion $f: M^{4 t} \rightarrow \mathbb{R}^{6 t}$, and project it to two nonparallel hyperplanes, then we get a map $f^{\prime}: M^{4 t} \rightarrow \mathbb{R}^{6 t-2}$. Let us denote the two hyperplanes $H_{1}, H_{2}$. The projection of $f$ to $H_{i}$ shall be called $f_{i}$. It is obvious that those and only those points belong to $\Sigma^{2} f^{\prime}$ which belong to $\Sigma^{1} f_{1}$ and $\Sigma^{1} f_{2}$ at the same time. This means that for this $f^{\prime}$ we have $\left[\Sigma^{2} f^{\prime}\right]=\left[\Sigma^{1} f_{1}\right] \cup\left[\Sigma^{1} f_{2}\right]$. The two cohomology classes on the right are both equal to the Thom polynomial of the $\Sigma^{1}$ singularity, which is the Euler class of the normal bundle of $f$ (see eg Szúcs [8]). As this normal bundle has rank $2 t$, the square of its Euler class is equal to $p_{t}\left(v_{f}\right)$, which is the same as $p_{t}\left(v_{M}\right)$. Thus we have proved our claim for those maps $M^{4 t} \rightarrow \mathbb{R}^{6 t-2}$ where the source manifold can be immersed into $\mathbb{R}^{6 t}$.

Let us recall that by $\operatorname{Imm}^{\mathrm{SO}}(4 t, 2 t)$ we denote the cobordism group of oriented immersions from $4 t$-dimensional manifolds to $\mathbb{R}^{6 t}$. There is the natural forgetful map 
$\psi: \mathrm{Imm}^{\mathrm{SO}}(4 t, 2 t) \rightarrow \Omega_{4 t}$ taking the cobordism class of an immersion to that of its underlying manifold. To finish the proof of the theorem it is sufficient to show, that this map has finite cokernel. According to the Pontrjagin-Thom construction and Serre's theorem on the stable Hurewicz homomorphism

and

$$
\operatorname{Imm}^{\mathrm{SO}}(4 t, 2 t) \cong \pi_{6 t}^{S} M \mathrm{SO}(2 t) \stackrel{\mathbb{Q}}{\cong} H_{6 t}(M \mathrm{SO}(2 t))
$$

where $\stackrel{\mathbb{Q}}{\cong}$ means "isomorphic if tensored with $\mathbb{Q}$ ". The forgetful map composed with these isomorphisms is clearly

$$
i_{*}: H_{6 t}(M \mathrm{SO}(2 t)) \rightarrow H_{4 t}(M \mathrm{SO})
$$

where $i: M \mathrm{SO}(2 t) \hookrightarrow M \mathrm{SO}$ is the natural inclusion. (Since $M \mathrm{SO}$ is not a space but a spectrum the meaning of "the inclusion $i: M \mathrm{SO}(2 t) \rightarrow M \mathrm{SO}$ " needs an explanation. We mean here that the homomorphism $i_{*}$ is induced by the inclusion $S^{N} M \mathrm{SO}(2 t) \rightarrow M \mathrm{SO}(2 t+N)$ composed - from the left and the right - with the isomorphisms $H_{6 t}\left(M \mathrm{SO}(2 t) \approx H_{6 t+N}\left(S^{N} M \mathrm{SO}(2 t)\right)\right.$ and $H_{6 t+N}(M \operatorname{SO}(2 t+N)) \approx$ $H_{4 t}(M S O)$.) Thus $\psi$ has finite cokernel if and only if

$$
i_{*} \otimes \mathbb{Q}: H_{6 t}(M \operatorname{SO}(2 t) ; \mathbb{Q}) \rightarrow H_{4 t}(M \mathrm{SO} ; \mathbb{Q})
$$

is epimorphic. The latter is equivalent to (by taking the dual morphism in cohomology)

$$
\left.i^{*}: H^{4 t}(M \mathrm{SO} ; \mathbb{Q}) \rightarrow H^{6 t}(M \operatorname{SO}(2 t) ; \mathbb{Q})\right)
$$

being monomorphic. We can apply the Thom isomorphism to further reduce the problem to showing that

$$
j^{*}: H^{4 t}(B \mathrm{SO} ; \mathbb{Q}) \rightarrow H^{4 t}(B \mathrm{SO}(2 t) ; \mathbb{Q})
$$

is monomorphic. Here $j: B \mathrm{SO}(2 t) \hookrightarrow B \mathrm{SO}$ is the natural inclusion map. The cohomology ring of $B \mathrm{SO}(2 t)$ is the polynomial ring $\mathbb{Q}\left[p_{1}, p_{2}, \ldots, p_{t-1}, \chi_{2 t}\right]$ generated by the Pontrjagin classes and the Euler class, whose square is $p_{t}$. On the other hand $H^{*}(B S O ; \mathbb{Q}) \cong \mathbb{Q}\left[p_{1}, p_{2}, \ldots\right]$. As $j$ takes each Pontrjagin class to the same Pontrjagin class, we get that $j^{*}$ is indeed injective in dimension $4 t$. This completes the proof of $\mathrm{tp}_{\Sigma^{2}}=p_{t}$.

The proof of the next theorem copies the proof of the previous section. The equality below in the Theorem is meant in the cohomology groups with rational coefficients. 
Theorem 5.5 Let $f: M_{1}^{n_{1}} \rightarrow N^{n_{1}+k_{1}}, g: M_{2}^{n_{2}} \rightarrow N_{2}^{n_{2}+k_{2}}$ be two generic maps of even codimension. Then for a generic perturbation of their product we have

$\left[\Sigma^{2}(f \times g)\right]=\sum_{j \geq 1}\left(\left[\Sigma^{2} f_{2 j-2}\right] \times\left(\operatorname{id}_{M_{2}}^{2 j}\right)^{*}\left[\Sigma^{2} g_{(-2 j)}\right]+\left(\operatorname{id}_{M_{1}}^{2 j}\right)^{*}\left[\Sigma^{2} f_{(-2 j)}\right] \times\left[\Sigma^{2} g_{2 j-2}\right]\right)$.

\section{References}

[1] V I Arnol'd, V A Vasiliev, V V Goryunov, O V Lyashko, Singularities. I. Local and global theory, from: "Dynamical systems VI", (R V Gamkrelidze, editor), Encycl. Math. Sci. 6, Springer, Berlin (1993) 237pp MR1088739

[2] G Braun, G Lippner, Characteristic numbers of multiple-point manifolds, Bull. London Math. Soc. 38 (2006) 667-678 MR2250759

[3] Y Byun, S Yi, Product formula for self-intersection numbers, Pacific J. Math. 200 (2001) 313-330 MR1868694

[4] P J Eccles, M Grant, Bordism groups of immersions and classes represented by selfintersections, Algebr. Geom. Topol. 7 (2007) 1081-1097 MR2336250

[5] R Rimányi, A Szúcs, Pontrjagin-Thom-type construction for maps with singularities, Topology 37 (1998) 1177-1191 MR1632908

[6] F Ronga, Le calcul de la classe de cohomologie entière duale à $\bar{\Sigma}^{k}$, from: "Proceedings of Liverpool Singularities-Symposium, I (1969/70)", Lecture Notes in Math. 192, Springer, Berlin (1971) 313-315 MR0293648

[7] A Szúcs, On the cobordism groups of immersions and embeddings, Math. Proc. Cambridge Philos. Soc. 109 (1991) 343-349 MR1085401

[8] A Szúcs, On the singularities of hyperplane projections of immersions, Bull. London Math. Soc. 32 (2000) 364-374 MR1750481

[9] A Szúcs, Cobordism of singular maps, Geom. Topol. 12 (2008) 2379-2452 MR2443969

[10] R Thom, Les singularités des applications différentiables, Ann. Inst. Fourier, Grenoble 6 (1955-1956) 43-87 MR0087149

Department of Mathematics, Harvard University

One Oxford Street, Cambridge 02138, United States

Department of Analysis, Eotvos University

Pazmany Peter setany 1/c, Budapest, 1117, Hungary

lipi@renyi.hu, szucs@cs.elte.hu

Received: 8 August 2008 Revised: 19 May 2010 